\title{
Performance of 3GPP LTE-Advanced Networks with Type I Relay Nodes
}

\author{
Zhangchao Ma, Yi Zhang, Kan Zheng, Wenbo Wang, Muzi Wu \\ Wireless Signal Processing and Network Lab \\ Key laboratory of Universal Wireless Communication, Ministry of Education \\ Beijing University of Posts \& Telecomms, Beijing, China \\ Email: mzc.bupt@gmail.com
}

\begin{abstract}
Relaying is expected to be one key element of the future cellular networks. This paper focuses on 3GPP LTEAdvanced network incorporated with Type I relay nodes. We first present the required radio resource management (RRM) framework which includes routing, resource partition and scheduling functions. Then two RRM strategies are suggested: one with the conventional cellular settings, the other with further consideration on the special interference environment with relay nodes (RN). We evaluate and compare the network performances through system-level simulations under different relay deployment patterns. The results show that the interferenceaware scheme could boost the coverage range of the $R N$ and provide better cell-edge throughput when the RN density is low. Moreover, when the RN number increased, a bottleneck effect caused by the limited backhaul link capacity is observed.
\end{abstract}

\section{INTRODUCTION}

Relaying has been understood as one of the most promising techniques towards the next generation of mobile communication system. It has great potential of further boosting the cellular network's capacity and coverage. The 3rd Generation Partnership Project (3GPP) has previously tried to standardize relaying through the ad-hoc peer-to-peer multihop protocol, known as opportunity-driven multiple-access (ODMA) technique [1]. The user equipments (UE) at cell edge are enabled to communicate with the BS through the help of the other UEs closer to the BS in a multihop way. However the heavy complexity and overhead required made this a failed approach. Being more practical and feasible, the infrastructure-based relay has become a more attractive approach. As observed in [2], the deployment of the new network element-relay node, could bring in noticeable reduction in CAPEX (capital expenditure, e.g. equipment cost) and OPEX (operational expenditure, i.e., operation \& maintenance, site rent etc.).

LTE-Advanced, as a major enhancement of 3GPP Long Term Evolution (LTE) standard, has defined two types of relay nodes [3]. Type I relay can transmit its own control signals. It acts just like a new BS (termed as eNB (evolved node B) in $3 \mathrm{GPP}$ ) to the UE, with the objective of coverage extension and capacity improvement. Type II relay doesn't has own control signals. It is transparent to the UEs, with the main objective to assist and enhance the donor eNB's signal.

In this paper, we focus on the former type of RN. Although Type I RN merely forwards data through the layer 3 and is noted for good compatibility, it still poses new problems,

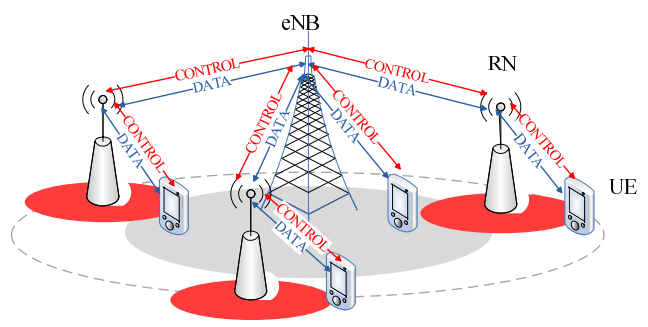

Fig. 1. Illustration of network model

e.g. routing, resource partition. We manifest the roles and functionalities of these key functions within the proposed RRM framework for cellular relay network. The performance of relaying is affected by the collaborative resource allocation strategies. We design two strategies based on the framework: one with the conventional cellular setting, i.e. power-based routing and full resource reuse, and the other with concern on the special interference environment with relay nodes. We build system-level simulation platform to evaluate and compare the network performances. Simulation results are obtained for scenarios with different deployment pattern of relay nodes.

In the rest of this paper, we first describe the system with Type I RN in section II, then we illustrate the RRM framework and the two suggested resource allocation strategy in section III. The simulation results are demonstrated in section IV, and finally our conclusions are made in section V.

\section{SySTEM DESCRIPTION}

According to 3GPP LTE-Advanced technical report [3], Type I RN has its own cell identifier. It is able to transmit its own synchronization channel, reference signals and physical control channels. It could be viewed as a full-functional eNB, except that it has to use a wireless backhaul to connect to the radio-access network via the donor cell. From a legacy UE's perspective, there will be no difference between the cell controlled by a Type I RN and the cell controlled by a normal eNB. The UEs could either connected to an eNB, called the macro UE, or connected to a $\mathrm{RN}$, called the relay UE. But no cooperative work between $\mathrm{eNB}$ and $\mathrm{RN}$ is considered to reduce complexity. 


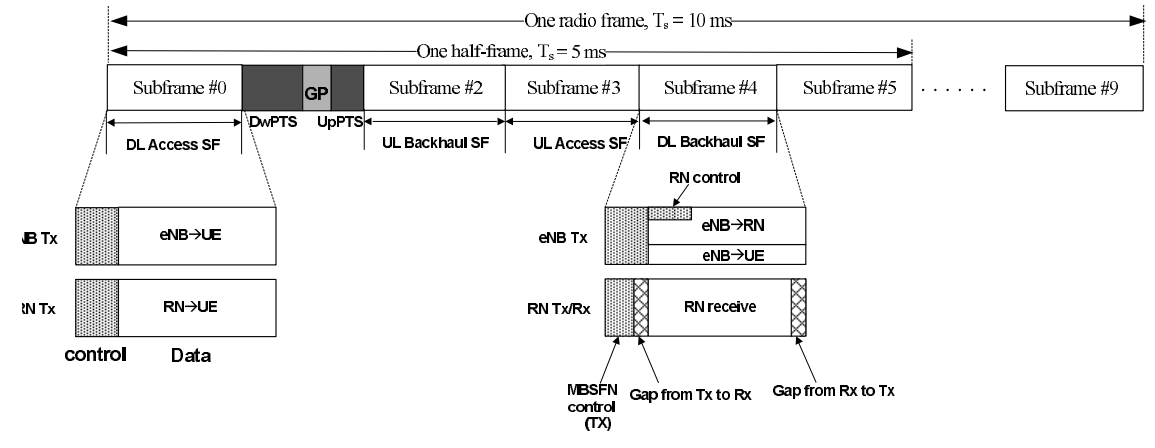

Fig. 2. Frame Structure

For the relay UE, only two hop relay transmission is supported. The data packets are relayed and forwarded in the layer 3. Thus the RN-UE interface could maintain perfect backward-compatibility since there is no difference from the legacy eNB-UE interface. For the backhaul link, i.e. the eNB$\mathrm{RN}$ interface, Type I relay shares the same frequency as the RN-UE link, known as in-band relaying. Moreover, the eNB$\mathrm{RN}$ and RN-UE links are time division multiplexed in order to avoid self-interference. As shown in Fig. 2, for every $10 \mathrm{~ms}$ radio frame, the $1 \mathrm{~ms}$ subframes can only be assigned to either backhaul or access links (eNB/RN-UE). Note that for the TDD frame structure, the $0,1,5$, 6th subframes are customized to carry synchronization channels, and thus not available for the backhaul link. Moreover, the legacy UE will regularly receive and measure the reference signals (RS) for each subframe. In order to avoid measurement error, the backhaul subframes are configured as MBSFN ones [4], then the legacy UE will ignore the RS during these backhaul "gaps". Although backwardcompatibility is obtained in this way, the available OFDM symbols for the eNB-RN transmission are reduced for more overhead (10 OFDM symbols is assumed in this study).

\section{Radio Resource Management}

\section{A. Framework Description}

Different from the eNB only network, the radio resource management for the REC network involves new aspects: user routing (i.e., determine whether to relay and select which relay node to go through), and multi-hop scheduling (i.e, determine resource block mapping for users and their respective links). The optimal solution would require joint processing [5], and global knowledge of the interference and channel state information of the whole network should be collected to a central control-unit. This will result in great amount of signaling overhead, complexity and delay. However, Type I relay is designed for decentralized network, where the eNB and RN are able to perform scheduling independently based on local information only. And the routing and scheduling functions are decoupled to reduce complexity. In the following we'll illustrate the three key functions that should be included in the decentralized RRM framework: routing, resource partition and packet scheduling.
1) Routing: Supposing there are several relay nodes surrounding the relay UE, with their channels'qualities fluctuating on the air, this UE could select the best relay according to instantaneous channel states and obtain diversity gain through opportunistic routing [6]. However, just like the eNB, the association between Type I relay and UE can not vary as frequently as possible to chase the instantaneous channel fading. More practically, the user routing should be based on long-term averaged channel quality measurement and stay unchanged until the serving signal quality dropped to a dedicated level, just like a normal handover.

2) Resource partition: Due to the in-band backhaul, the radio resource should be partitioned for access and backhaul link. For Type I relay system, this is done through allocation of MBSFN frame, which is controlled through high layer signals semi-statically. The system can estimate the capacity of the RNs' access link and backhaul link, then decide the subframe allocation pattern which could match them closely. Furthermore, the subframes marked as access link could be partitioned further into certain resource spaces for different RNs and eNB to perform scheduling. Through adjusting the size and location of these resource spaces, the system could achieve fairness maintenance or interference avoidance between different nodes.

3) Scheduling: In Type I relay system, the access link and backhaul link scheduling are performed independently. The mature adaptive transmission techniques such as AMC and HARQ could be inherited to exploit the channel diversity. For the access link, Type I RN would just act like a normal eNB. It could schedule its connected relay UEs with ordinary channel-aware algorithms, such as proportional-fair (PF), with the normal CQI feedback required. For the backhaul link, the ideal eNB-RN scheduling should guarantee the amount of data relayed to the $\mathrm{RN}$ just matching with the data required on the access link for each relay UE. However, the eNB can't obtain the exactly RN-UE side information in a decentralized Type I relay system. Supposing one of the relay UE has a weak access link, if the eNB keeps feeding more data to the RN, then its queue at the RN could be overflowed; moreover, if a handover is triggered, the data transferred to the $\mathrm{RN}$ has to be discarded and the consumed resources are wasted. Another case is that when a UE's access link becomes much better; 
however, it can't make full use of it if the backhaul link doesn't supply enough data. Thus, certain control mechanisms should be implemented to schedule the backhaul link on the UE level in order to avoid the UE service queues at the RN being overflowed or starved.

Herein we adopt a request-and-send mechanism to adjust the priority of different UEs. For downlink, a data-request signal will be triggered from the RN if the queue length of UE $k$ at $\mathrm{RN}$ is below a specific threshold, which is according to the estimated throughput achievable on the access link, i.e.:

$$
L\left(Q_{R N}^{k}\right)<\alpha \cdot C_{A}
$$

where $L()$ denote the current queue length, $C_{A}$ denote the estimation of access link capacity, and $\alpha$ is a threshold control variable which is less than one. Then in the following backhaul subframe, the eNB will only allocate resources for the UEs requested data in advance. Additionally, the unallocated resources on the backhaul link can be assigned to the macro UEs to improve resource utilization.

\section{B. Two Specific Resource Allocation Strategies}

Based on the given RRM framework, we envision two possible RRM schemes: in the first scheme all RNs and eNB simply reuse the resource on their access link and in the other scheme the RN and eNB would use orthogonal resource to avoid interference. We would detail the two schemes as below and present their performance comparison in section IV.

1) Scheme I: In this scheme, the conventional settings for a non-relay 3GPP LTE system are preserved. All the relay cells, treated as specific eNBs, could fully reuse the in-band frequency resource with the other normal eNBs. Moreover, the RSRP (reference signal received power) measurement is taken to determine whether a user will connect to a RN, i.e.:

$$
r_{s}=\arg \max \left\{P_{B}, P_{R}\right\}
$$

where $P_{B}$ and $P_{R}$ represent the received power from the best eNB and RN candidate. It could be easily proved that it is equivalent to the SINR (signal to interference-and-noise ratio) metric for the reuse 1 case, because:

$$
\begin{aligned}
& S I N R_{B}=\frac{P_{B}}{P_{B, \text { other }}+P_{R, \text { all }}+N}=\frac{P_{B}}{P_{\text {all }}-P_{B}} \\
& S I N R_{R}=\frac{P_{R}}{P_{B, \text { all }}+P_{R, \text { other }}+N}=\frac{P_{R}}{P_{\text {all }}-P_{R}}
\end{aligned}
$$

where $S I N R_{B}$ and $S I N R_{R}$ represent the signal SINR from the eNB and RN, respectively, $P_{x, \text { all }}, P_{x, \text { other }}$ represents the total received power and the power except serving signal from the eNBs or RNs, respectively, and $N$ is the variance of noise power. Then, there is:

$$
\arg \max \left\{P_{B}, P_{R}\right\} \Leftrightarrow \arg \max \left\{S I N R_{B}, S I N R_{R}\right\}
$$

2) Scheme II: Although the Type I RN is equipped with similar functions as the eNB, it has its own special features. According to [3], the $\mathrm{RN}$ is expected to be with low transmit power (e.g. $30 \mathrm{dBm}$ for relay vs. $46 \mathrm{dBm}$ for base), and low antenna height (e.g. $5 \mathrm{~m}$ for relay vs. $25 \mathrm{~m}$ for base), possibly

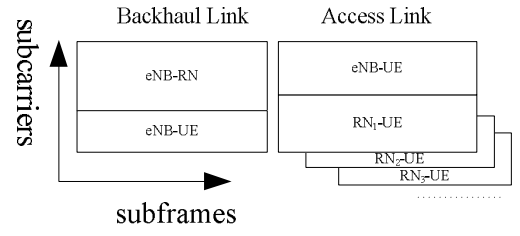

Fig. 3. Resource partition pattern for scheme II

mounted on the lamp posts to reduce cost. Considering these constraints, the signal from relay would attenuate much faster than the signal from an eNB. And this case would become even worse if the relay signal encounters the strong interference from the eNB. Thus, in this scheme the access link resources are further partitioned for the eNB and RNs as shown in Fig. 3. Then the eNB signal only interferes with the other eNBs, and the RN signal only interferes with the other RNs. Because the inter-relay interference is relatively small if they are deployed properly with enough geographic isolation, the resulting relay signal quality reflected in the SINR can be boosted. Thereby an interference-aware routing scheme should be employed to make use of this advantage, e.g.:

$$
r_{s}=\arg \max \left\{S I N R_{B}, S I N R_{R}\right\}
$$

where

$$
S I N R_{B}=\frac{P_{B}}{P_{B, \text { other }}+N}, S I N R_{R}=\frac{P_{R}}{P_{R, \text { other }}+N}
$$

Moreover, this partition work could consider the load of RN and eNB to impose some fairness between the relay UE and macro UE. Herein we simply partition the access link resource according to the number of users connected to the eNB and the RNs, i.e. : $N_{B, \text { access }} / N_{R, \text { access }}=K_{B} / K_{R}$, where $N_{B \text {,access }}$ and $N_{B, \text { access }}$ are the allocated resource blocks for the eNB and RNs, respectively. $K_{B}$ and $K_{R}$ are the number of macro UE and relay UE, respectively.

\section{Simulation Results}

We establish a dynamic system-level simulation platform to evaluate the performance of the LTE-Advanced network incorporated with Type I relay nodes. The network model consists of 19 macro eNBs, each with 3-sector antennas, and a constant number of relay nodes are deployed within each sector. The UEs are evenly distributed across the network, with the average number of 25 per sector. We consider two configurations for the deployment of relay nodes: the light pattern, where only one $1 \mathrm{RN}$ is deployed on the angle-bisector of each sector at a distance of half the inter-site distance (ISD) from the base station; and the dense pattern, where $10 \mathrm{RNs}$ per sector is deployed in a two-ties way as described in [7]. Given the initial positions, each RN is further adjusted in order to achieve larger LOS probability and overcome shadowing effect. This is the so-called site-planning technique [8], i.e., the dedicated RN's position is chosen from five randomly chosen candidates surrounding the initial position according to the backhaul SINR. Furthermore, the RNs are equipped with 
TABLE I

PARAMETERS ASSUMPTION

\begin{tabular}{l|l}
\hline Parameters & Values \\
\hline Inter-Site Distance (ISD) & $1732 \mathrm{~m}$ \\
\hline Bandwidth / Carrier & $10 \mathrm{MHz} / 2 \mathrm{GHz}$ \\
\hline Pathloss & according to TR36.814[3] \\
\hline Lognormal Shadowing & eNB/RN-UE:8dB/10dB std dev, \\
& $50 \mathrm{~m}$ correlation distance, \\
& 0.5 correlation between sites \\
\hline Fast fading model & eNB/RN $\rightarrow$ UE:SCME \\
& eNB $\rightarrow$ RN:SUI-1 \\
\hline Antenna configuration & eNB/RN/UE: $2 \times 2 \times 2$ multiplexing \\
\hline Transmit Power & eNB/RN : 46dBm/30dBm \\
\hline Antenna gain & eNB: $14 \mathrm{dBi}$ \\
& RN access/backhaul: $5 \mathrm{dBi} / 7 \mathrm{dBi}$ \\
\hline Antenna pattern & eNB : 70 ${ }^{\circ}$ directional \\
& RN : Omni-directional \\
\hline Penetration Loss & eNB/RN-UE:20dB \\
\hline Thermal Noise Density & $-174 \mathrm{dBm} / \mathrm{Hz}$ \\
\hline Noise Figure & $9 \mathrm{~dB}$ at UE, $5 \mathrm{~dB}$ at RN \\
\hline Scheduling algorithm & Proportional fair in time and frequency \\
\hline Traffic model & Full buffer \\
\hline AMC & 29 levels according to [9] \\
\hline HARQ & $10 \mathrm{~ms} \mathrm{RTT,} \mathrm{maximum} \mathrm{4} \mathrm{transmission} \mathrm{times}$ \\
\hline L-S interface & EESM \\
\hline &
\end{tabular}

$70^{\circ}$ directional antenna which always pointed to its donor eNB in order to provide better backhaul quality through interference reduction. The resource partition pattern for backhaul/access link is as shown in Fig. 2, i.e., at the ratio of 1:1. The main assumptions and parameters are shown in Table. I, which is mainly according to the methodology given in [3].

First, we compare the downlink geometry of the relay system with two proposed resource allocation schemes, and the system without relay is also taken as a reference in Fig. 4. It is shown that Scheme II could achieve better geometry performance compared to Scheme I. Especially for the light relay pattern (1RN/sec), Scheme II provides obvious improvement while Scheme I has little influence on the SINR distribution.

Further the access link SINR is displayed geometrically in Fig. 5. It could be observed that for the light relay pattern, the area with a improved SINR surrounding the RN is significantly enlarged compared to Scheme I. This validates the advantage of access-zone resource partition and SINR-based routing scheme, which could improve the relay signal's effect range. However, it is shown that under the dense relay pattern, this range expansion effect with Scheme II is not as significant as that in the light pattern, because the inter-relay interference is elevated with denser deployment. Through the relay/macro user ratio statistics in Fig. 6, it is also confirmed that under the light pattern, the relay UE ratio with Scheme II is almost three fold of that with Scheme I; while under the dense RN pattern, the relay UE ratio is almost the same for both Scheme I and Scheme II.

The user throughput CDF curves are illustrated in Fig.
7. It is shown that under the light relay pattern, the user throughput with Scheme I is only improved at the high level, while most users especially for the cell-edge UE (5\% CDF) experienced even deteriorated throughputs compared to the non-relay system. This is because with Scheme I only a small portion of users could get access to the RN and enjoy better signal; nevertheless, these users could reuse the whole access link resource with the major macro UEs, and thus achieve extremely high throughputs. However, the resources left for the major macro UEs are much less because that a large part of the backhaul link resources are consumed by the relay UE. It is also observed that with Scheme II all UEs including the celledge UEs obtains improved throughput, this is benefited from larger relay UE ratio and resource coordination. But when the RNs are densely deployed the situation changes. Now there are about $50 \%$ or relay UEs, i.e., most cell edge users could get access to RN either with Scheme I or Scheme II, and the remnant macro UEs are mostly located at the cell-center area. Thus it is observed that the overall user throughput is elevated over the non-relay case for both Scheme I and Scheme II. Moreover, the throughput improvement with Scheme I even outperforms Scheme II. This is because the SINR gain with Scheme II can not compromise with the reduction of available resources after further partition.

The aggregated system throughput statistics is further given in Fig. 8. The first two columns indicate the throughput achieved by macro UE in the backhaul and access subframes, respectively; the third column represents the relay UE throughput, while the last column is the aggregated cell throughput. It is shown that with the RN number increased, the backhaul resources are drained by the relay UE. It is observed that the macro UE throughput at backhaul subframe is nearly zero under the dense RN pattern. Correspondingly, the throughput of relay UEs meets its bottleneck when the RN density increased. The macro UE throughput in access subframes with Scheme II is always lower than Scheme I, for there are less available resource and the macro signal is less sensitive to the interference from the RNs. Thus Scheme I shows higher aggregated throughput compared to Scheme II. The cell throughput with RNs could achieve prominently $40 \% \sim 70 \%$ gain from the non-relay system, which is mainly contributed from the good backhaul link quality.

\section{CONClusion}

This paper presents the radio resource management framework for LTE-Advanced network with Type I relay nodes, which are featured with low power, low height nodes, and with independent scheduling functions. In addition to the conventional resource management strategy, we propose one eNB/RN resource partition scheme to deal with the special interference environment when relay nodes are introduced. The performance of such a network is studied by systemlevel simulation. It shows that the impact of relaying to the network performance is sensitive to the resource allocation strategies and relay configurations. With low RN density, the interference-aware scheme shows greater $\mathrm{RN}$ coverage range 


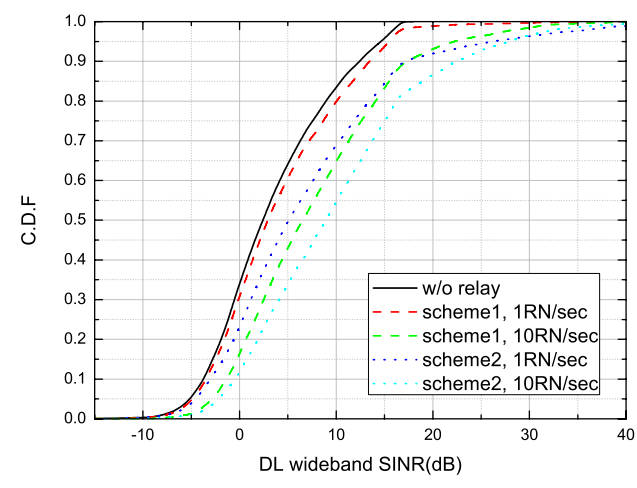

Fig. 4. Comparison of downlink wideband SINR CDF curves
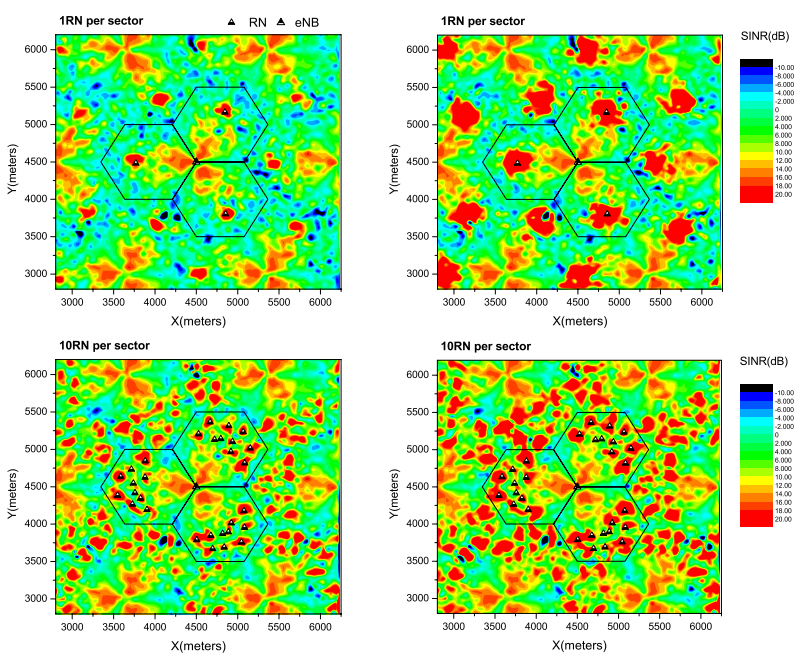

Fig. 5. Comparison of geometric SINR distributions

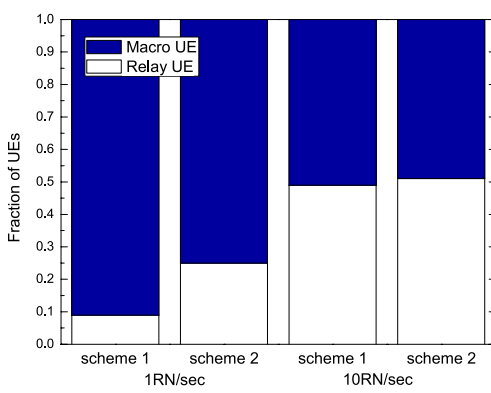

Fig. 6. Comparison of macro/relay UE ratio

especially and provide better cell-edge throughput. However, when the RNs are deployed densely enough, the range expansion benefit is compromised while the spectral efficiency can be decreased due to the partitioned resource. Moreover, the gain from relaying can not keep growing with the RN number increasing. It will be finally limited at the backhaul link capacity.

\section{ACKNOWLEDGMENT}

This work was supported in part by the China NSFC under Grant 60802082, and National Key Technology R\&D Program

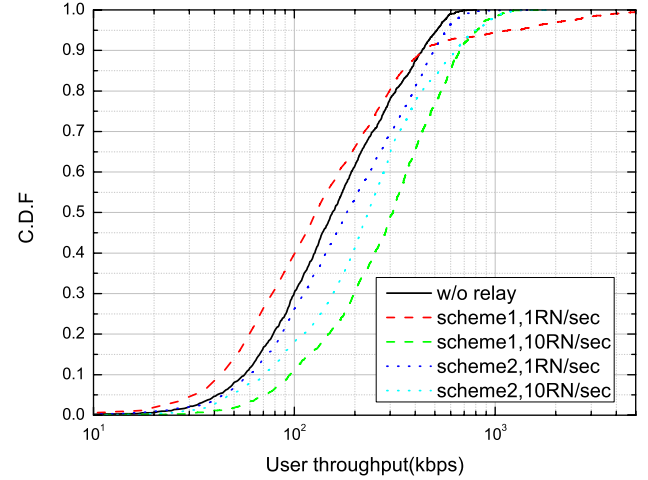

Fig. 7. Comparison of user throughput CDF curves

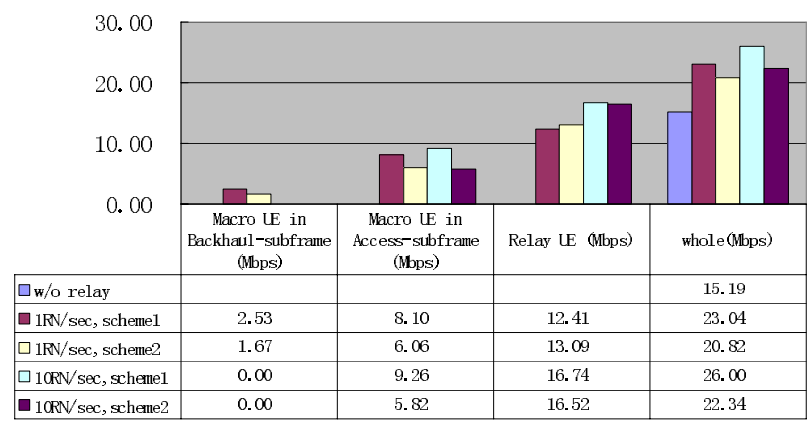

Fig. 8. Comparison of aggregated system throughput

of China under Grant 2009ZX03003-004-01.

\section{REFERENCES}

[1] T. Rouse, I. Band, and S. McLaughlin, "Capacity and power investigation of opportunity driven multiple access (ODMA) networks in TDD-CDMA based systems," IEEE International Conference on Communications, pp. 3202-3206, May 2002.

[2] E. Lang, S. Redana, and B. Raaf, "Business Impact of Relay Deployment for Coverage Extension in 3GPP LTE-Advanced," ICC '09, LTE Evolution Wksp., June 14-18, 2009.

[3] 3GPP TR 36.814 v1.0.1, "3rd Generation Partnership Project; Technical Specification Group Radio Access Network; Further Advancements for E-UTRA Physical Layer Aspects," Sep. 2008.

[4] 3GPP R1-091689, "MBSFN Subframe and Control Structure for Relay Type 1 nodes", NEC Group, RAN1\#57, May. 2009.

[5] H. Viswanathan, and S. Mukherjee, "Performance of cellular networks with relays and centralized scheduling," IEEE Transactions on Wireless Communications, vol. 4, issue. 5, pp. 2318-2328, Sept. 2005.

[6] Aggelos B., Hyundong S., Moe Z.W., and Andrew L. "Cooperative diversity with opportunistic relaying", IEEE WCNCO6, pp. 1034-1039, Sept. 2006

[7] 3GPP R1-093562, "Relay System level simulation", China Mobile, RAN1\#58, Aug. 2009.

[8] 3GPP R1-093726, "Text Proposal for Channel Model and Evaluation Methodology", China Mobile, RAN1\#58, Aug. 2009.

[9] 3GPP TS 36.211 V8.2.0, "3rd Generation Partnership Project Technical Specification Group Radio Access Network Evolved Universal Terrestrial Radio Access (E-UTRA) Physical Channels and Modulation (Release 8)," Mar. 2008. 